\title{
X-rays from the mode-switching PSR B0943+10
}

\author{
S. Mereghetti ${ }^{1}$, L. Kuiper ${ }^{2}$, A. Tiengo ${ }^{3,1,4}$, J. Hessels ${ }^{5,9}$, \\ W. Hermsen ${ }^{2,9}$, K. Stovall ${ }^{6}$, A. Possenti ${ }^{7}$, J. Rankin ${ }^{8}$, P. Esposito ${ }^{9}$, \\ R. Turolla ${ }^{10,11}$, D. Mitra ${ }^{8,12,13}$, G. Wright ${ }^{14}$, B. Stappers ${ }^{14}$, \\ A. Horneffer ${ }^{15}$, S. Oslowski ${ }^{15,16,17}$, M. Serylak ${ }^{18,19}$, \\ J.-M. Griessmeier ${ }^{20,19}$, M. Rigoselli ${ }^{1,21}$ \\ 1 INAF-IASF Milano, Italy \\ ${ }^{2}$ SRON, Utrecht, The Netherlands \\ ${ }^{3}$ Scuola Universitaria Superiore IUSS Pavia, Italy \\ ${ }^{4}$ INFN, Sezione di Pavia, Italy \\ ${ }^{5}$ ASTRON, Dwingeloo, The Netherlands \\ ${ }^{6}$ Department of Physics and Astronomy, University of New Mexico, Albuquerque, NM, USA \\ ${ }^{7}$ INAF - Osservatorio Astronomico di Cagliari, Selargius, Italy \\ 8 Physics Department, University of Vermont, Burlington, VT 05405, USA \\ 9 Anton Pannekoek Institute for Astronomy, Univ. of Amsterdam, The Netherlands \\ 10 Dipart. di Fisica e Astronomia, Università di Padova, Italy \\ ${ }^{11}$ MSSL-UCL, Holmbury St. Mary, Dorking, UK \\ 12 National Centre for Radio Astrophysics, Ganeshkhind, Pune, India \\ 13 Janusz Gil Institute of Astronomy, Univ. of Zielona Góra, Poland \\ 14 Jodrell Bank Centre for Astrophysics, Univ. of Manchester, UK \\ ${ }^{15}$ Max-Planck-Institut für Radioastronomie, Bonn, Germany \\ ${ }^{16}$ Fakultät für Physik, Universität Bielefeld, Germany \\ 17 currently at Swinburne Univ. of Technology, Australia \\ 18 Dept. of Physics \& Astronomy, Univ. of the Western Cape, Bellville, South Africa \\ 19 Station de Radioastronomie de Nançay, Observatoire de Paris, CNRS, Nançay, France \\ 20 LPC2E - Université d'Orléans, France \\ ${ }^{21}$ Università di Milano Bicocca, Milano, Italy
}

\begin{abstract}
New simultaneous X-ray and radio observations of the archetypal mode-switching pulsar PSR B0943+10 have been carried out with XMM-Newton and the LOFAR, LWA and Arecibo radio telescopes in November 2014. They allowed us to better constrain the X-ray spectral and variability properties of this pulsar and to detect, for the first time, the X-ray pulsations also during the X-ray-fainter mode. The combined timing and spectral analysis indicates that unpulsed non-thermal emission, likely of magnetospheric origin, and pulsed thermal emission from a small polar cap are present during both radio modes and vary in a correlated way.
\end{abstract}

Keywords. pulsars: general, X-rays: individual (PSR B0943+10), stars: neutron

\section{Introduction}

Until recently, the study of variability in rotation-powered pulsars was limited to the radio band, where changes in the emission properties related to phenomena occurring on very different time scales, from sub-pulses to long-term moding and intermittence, are routinely observed. On the contrary, rotation-powered neutron stars were generally believed to have a steady luminosity at X- and $\gamma$-ray energies, to the point that they are typically used as calibration sources. Some X-ray variability, in the form of short bursts associated with X-ray flux enhancements and spectral changes, has been observed in two allegedly rotation-powered pulsars (Gavriil et al. 2008; Archibald et al. 2016), and some 
long term X-ray variations have also been observed in one member of the XDIN class (Hohle et al. 2012). However, all these variable phenomena are believed to result from magnetic activity and are not expected to occur in old radio pulsars with "canonical" magnetic fields (see, e.g., Mereghetti, Pons \& Melatos 2015).

The situation has changed with the recent discovery of variations in the X-ray flux of PSR B0943+10 (Hermsen et al. 2013), one of the mode-switching pulsars best studied in the radio band. Here, we briefly review the most important results obtained for PSR B0943+10 and compare its properties with those of other mode-switching pulsars recently observed at X-ray energies.
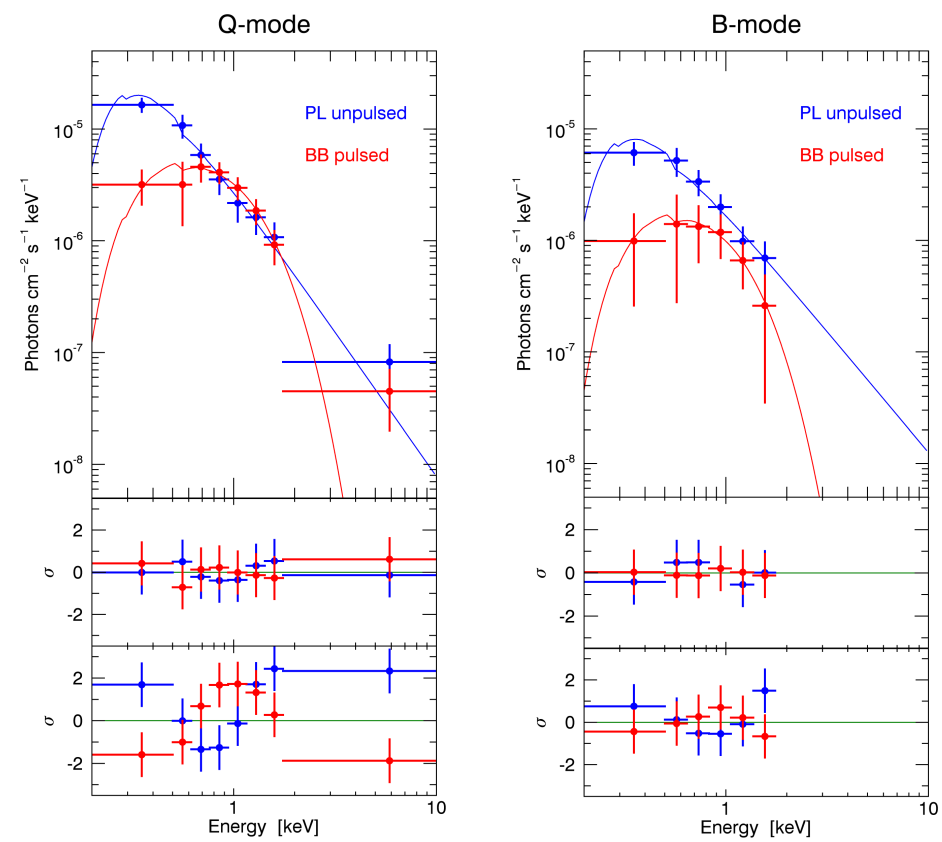

Figure 1. X-ray spectra of the unpulsed (blue) and pulsed (red) emission from PSR B0943+10 in the Q-mode (left) and B-mode (right). In the top panels the unpulsed flux is fitted with a power law and the pulsed flux with a blackbody. The corresponding residuals are shown in the middle panels. The bottom panels, which show the residuals obtained by fitting the unpulsed flux with a blackbody and the pulsed flux with a power law, demonstrate that this fit is acceptable for the B-mode but not for the Q-mode.

\section{X-ray results}

PSR B0943+10 $\left(P=1.1 \mathrm{~s}, \dot{P}=3.5 \times 10^{-15} \mathrm{~s} \mathrm{~s}^{-1}\right)$ is a relatively old pulsar $(\tau=5 \mathrm{Myr})$ with a dipole magnetic field of $4 \times 10^{12} \mathrm{G}$. In the radio band, at irregular intervals of $\lesssim$ few hours, it alternates between a radio bright (B-) mode, characterized by regularly drifting subpulses, and a quiescent (Q-) mode in which the radio emission is on average fainter and the subpulses have a chaotic pattern (Suleimanova \& Izvekova 1984; Bilous 2017).

The faint X-ray emission from PSR B0943+10 (few $10^{-15} \mathrm{erg} \mathrm{cm}^{-2} \mathrm{~s}^{-1}$ ) was discovered in 2003 (Zhang et al. 2005), but it could be studied in detail only when longer observations with simultaneous radio coverage were obtained in 2011. This made it possible to analyse separately the X-ray data of the two radio modes, leading to the discovery of X-ray 
Table 1. Comparison of three mode-switching pulsars observed in X-rays

\begin{tabular}{|c|c|c|c|}
\hline | & PSR B0943+10 & I PSR B0823+26 & PSR B1822-09 \\
\hline $\mid \begin{array}{l}P[\mathrm{~s}] \\
\dot{P}\left[\mathrm{~s} \mathrm{~s}{ }^{-1}\right] \\
\tau[\mathrm{Myr}] \\
B[\mathrm{G}] \\
\dot{E}_{\text {rot }}\left[\mathrm{erg} \mathrm{s}^{-1}\right]\end{array}$ & $\begin{array}{c}1.1 \\
3.5 \times 10^{-15} \\
5 \\
2 \times 10^{12} \\
10^{32}\end{array}$ & $\begin{array}{c}0.53 \\
1.7 \times 10^{-15} \\
5 \\
9.6 \times 10^{11} \\
4.5 \times 10^{32}\end{array}$ & $\begin{array}{c}0.77 \\
5.3 \times 10^{-14} \\
0.2 \\
6.5 \times 10^{12} \\
4.6 \times 10^{33}\end{array}$ \\
\hline | Distance [kpc] & 0.89 & 0.31 & 0.26 \\
\hline | Likely geometry & aligned & 〜orthogonal & $\sim$ orthogonal \\
\hline | Mode duration & $\sim$ hours & $\mid \sim$ hours (also nulling) $\mid$ & few minutes \\
\hline | Radio interpulse & No & Yes & Yes (in Q-mode) \\
\hline |Radio pre/postcursc & I precursor in Q-mod & | postcursor in B-mode | & in B-mode \\
\hline | Drifting subpulses & in B-mode & Yes & No \\
\hline X-ray flux & I brighter in Q-mode & | brighter in B-mode & constant \\
\hline$L_{X}\left[\mathrm{erg} \mathrm{s}^{-1}\right]$ & $\sim 2 \times 10^{29}$ & $\sim 2 \times 10^{28}$ & $\sim 3 \times 10^{29}$ \\
\hline Total X-ray spectru & $\mathrm{n} \mid \mathrm{BB}+\mathrm{PL}($ or $\mathrm{BB}+\mathrm{BB}$ & $\mid \mathrm{BB}+\mathrm{BB}($ or $\mathrm{BB}+\mathrm{PL}) \mid$ & $\mathrm{BB}+\mathrm{BB}$ \\
\hline $\begin{array}{l}\text { Spectral-timing } \\
\text { analysis }\end{array}$ & $\begin{array}{c}\text { BB pulsed } \\
\text { PL unpulsed }\end{array}$ & favors $\mathrm{BB}+\mathrm{BB}$ & both BB pulsed \\
\hline References & \multicolumn{3}{|c|}{ | Mereghetti et al. 2016| Hermsen et al. 2018 | Hermsen et al. 2017} \\
\hline
\end{tabular}

variability anti-correlated with the radio flux (Hermsen et al. 2013). It was found that, during the radio-fainter $\mathrm{Q}$-mode, the $\mathrm{X}$-ray flux is brighter by a factor $\sim 2.5$, shows X-ray pulsations, and has a blackbody plus power-law spectrum. In the B-mode, the spectrum was less constrained, being fit equally well by either a blackbody or a power law.

We performed a new observing campaign in November 2014, using XMM-Newton and the LOFAR, LWA and Arecibo radiotelescopes (Mereghetti et al. 2016), obtaining Xray esposures ( $\sim 120 \mathrm{ks}$ in Q- and $\sim 175 \mathrm{ks}$ B-mode) longer by factors of 2.4 and 3.5 than those of the previous data, respectively. This allowed us to better constrain the spectral properties of PSR B0943+10 and to detect its X-ray pulsations also during the $\mathrm{B}$-mode. The new data show that the phase-averaged spectrum is well fit by the sum of a power law with photon index $\sim 2.4$ and a blackbody in both radio modes. The blackbody temperatures in the two modes are $k T_{Q}=0.27 \pm 0.04 \mathrm{keV}$ and $k T_{B}=0.24 \pm 0.03 \mathrm{keV}$ and the emitting radii are of the order of $\sim 30 \mathrm{~m}$ (for $\mathrm{d}=890 \mathrm{pc}$ ).

By applying a maximum likelihood spectral analysis which used also the timing information of the detected counts, we derived the spectra of the pulsed and unpulsed emission. As shown in Fig. 1 (left), we confirmed that in the Q-mode the pulsed flux is thermal while the unpulsed flux is non-thermal. The blackbody describing the pulsed emission and the power law describing the unpulsed emission are consistent with the two components used to fit the total spectrum of the Q-mode.

The situation is less well constrained for the B-mode: for both the pulsed and unpulsed emission, it is impossible to distinguish between a power law and a blackbody, since they give similarly good fits (Fig. 1, right). However, it is reasonable to assume that the same two components seen in the Q-mode are present, although with a reduced flux, also during the B-mode. In other words, we attribute the pulsations to the thermal component in both modes. When the pulsar changes from the $\mathrm{Q}$ to the B-mode, the thermal flux decreases by a factor $\sim 3$ and the non-thermal one decreases by a factor $\sim 2$. 


\section{Discussion}

The results obtained in the 2014 campaign indicate that the X-ray properties of PSR B0943+10 in the two radio modes are not as different as it was previously thought: a blackbody plus power law spectrum and X-ray pulsations (which can be attributed to the thermal component) are present in both modes, although with different fluxes. Thus, it is not necessary to invoke global magnetospheric changes capable of entirely suppressing one of the spectral components (Hermsen et al. 2013; Mereghetti et al. 2013) when the pulsar switches to its X-ray fainter mode. The small emitting area inferred from the blackbody fits is broadly consistent with the polar cap size expected in the partially screened gap model, which is smaller than that of a pure dipole owing to multipolar field components (Gil et al. 2007).

The mechanism causing the mode-switching in radio pulsars is still unknown and the study of X-ray variability might help to understand the origin of this phenomenon. Indeed, the interesting results obtained for PSR B0943+10 prompted X-ray observations of other mode-switching pulsars. Unfortunately, these old and not very energetic pulsars are faint in X-rays. Up to now, XMM-Newton data have been obtained for PSR B1822-09 (Hermsen et al. 2017) and PSR B0823+26 (Hermsen et al. 2018). Their properties are compared with those of PSR B0943+10 in Table 1.

PSR B1822-09, younger and more energetic than PSR B0943+10, shows a main radio pulse and a radio interpulse separated by $\sim 0.5$ in phase and, contrary to PSR B0943 +10 , is believed to be a nearly orthogonal rotator. Its Q- and a B-mode radio states last only a few minutes and no evidence for X-ray variations was found. Its X-ray spectrum is fit by the sum of two blackbodies with $k T_{1}=0.08 \mathrm{keV}, k T_{2}=0.19 \mathrm{keV}$ and emitting radii $R_{1}=2 \mathrm{~km}, R_{2}=100 \mathrm{~m}$. Both the hotter and the cooler blackbody components seem to contribute to the X-ray pulsations, which are present in both radio modes. The lack of $\mathrm{X}$-ray variability could be due to the short duration of the radio modes. This could arise, e.g., if the timescale for the X-ray variation is longer than the mode duration. The timing parameters of PSR B0823+26 are very similar to those of PSR B0943+10. It also shows a bright and a quiet radio mode (Sobey et al. 2015), but, contrary to PSR B0943+10, the X-ray flux is higher during the B-mode.

Altough the global picture emerging from these data is still puzzling, the heterogeneous pattern of X-ray/radio variations seen in these few objects, anticipates that future, more sensitive observations will reveal a rich phenomenology that can potentially provide interesting clues on the mode-switching mechanism, and more in general, on the emission processes of rotation-powered pulsars.

\section{References}

Archibald, R. F., Kaspi, V. M., Tendulkar, S. P., Scholz, P. 2016, ApJ, 829, L21

Bilous, A. 2017, $A \& A$, in press

Gavriil, F. P., Gonzalez, M. E., Gotthelf, E. V., et al. 2008, Science, 319, 1802

Gil, J., Melikidze, G., Zhang, B. 2007, MNRAS 376, L67

Hermsen W. et al. 2013, Science, 339, 436

Hermsen W., Kuiper, L., Hessels, J.W.T., et al. 2017, MNRAS, 466, 1688

Hermsen W. et al. 2018, in preparation

Hohle, M. M., Haberl, F., Vink, J., et al. 2012, MNRAS, 423, 1194

Mereghetti, S. and Tiengo, A. and Esposito, P. and Turolla, R. 2013, MNRAS, 435, 2568

Mereghetti, S., Pons, J. A., Melatos, A. 2015, Space Sci. Revs, 191, 315

Mereghetti, S., Kuiper, L., Tiengo, A. et al. 2016, ApJ, 831, 21

Sobey, C. et al. 2015, MNRAS, 451, 2493

Suleimanova, S. A. \& Izvekova, V. A. 1984, Soviet Astron., 28, 32

Zhang, B., Sanwal, D., Pavlov, G. G. 2005, ApJ, 624, L109 